PROCEEDINGS OF THE

AMERICAN MATHEMATICAL SOCIETY

Volume 132, Number 11, Pages 3177-3187

S 0002-9939(04)07525-2

Article electronically published on May 12, 2004

\title{
CHARACTERIZING COHEN-MACAULAY LOCAL RINGS BY FROBENIUS MAPS
}

\author{
RYO TAKAHASHI AND YUJI YOSHINO
}

(Communicated by Bernd Ulrich)

\begin{abstract}
Let $R$ be a commutative noetherian local ring of prime characteristic. Denote by ${ }^{e} R$ the ring $R$ regarded as an $R$-algebra through $e$-times composition of the Frobenius map. Suppose that $R$ is F-finite, i.e., ${ }^{1} R$ is a finitely generated $R$-module. We prove that $R$ is Cohen-Macaulay if and only if the $R$-modules ${ }^{e} R$ have finite Cohen-Macaulay dimensions for infinitely many integers $e$.
\end{abstract}

\section{INTRODUCTION}

Throughout the present paper, we assume that all rings are commutative and noetherian.

Let $R$ be a local ring of characteristic $p$, and let $f: R \rightarrow R$ be the Frobenius map, given by $a \mapsto a^{p}$. For an integer $e$, we denote by $f^{e}: R \rightarrow R$ the $e$-th power of $f$; that is, it is given by $f^{e}(a)=a^{p^{e}}$. We denote by ${ }^{e} R$ the $R$-algebra $R$ whose $R$-algebra structure is given via $f^{e}$. The ring $R$ is said to be $F$-finite if ${ }^{1} R$, hence every ${ }^{e} R$, is a finitely generated $R$-module.

E. Kunz [18] proved that $R$ is regular if and only if ${ }^{1} R$ is $R$-flat. A. G. Rodicio 21] gave a generalization of this result as follows.

Theorem 1.1 (Rodicio). A local ring $R$ of prime characteristic is regular if and only if the $R$-module ${ }^{1} R$ has finite flat dimension.

A similar result concerning the complete intersection property and complete intersection dimension (abbr. CI-dimension) was proved by Blanco and Majadas 9]. They actually proved that a local ring $R$ of prime characteristic is a complete intersection if and only if there exists a Cohen factorization $R \rightarrow S \rightarrow \widehat{{ }^{1} R}$ such that the CI-dimension of $\widehat{1} R$ over $S$ is finite, where $\widehat{{ }^{1} R}$ denotes the completion of the local ring ${ }^{1} R$. (For the definition of a Cohen factorization, see [5].) Therefore, we have in particular the following.

Theorem 1.2 (Blanco and Majadas). An F-finite local ring $R$ is a complete intersection if and only if the $R$-module ${ }^{1} R$ has finite CI-dimension.

Received by the editors May 15, 2002 and, in revised form, April 9, 2003 and August 7, 2003. 2000 Mathematics Subject Classification. Primary 13A35, 13D05, 13H10.

Key words and phrases. Frobenius map, CM-dimension, G-dimension, flat dimension, injective dimension.

(C)2004 American Mathematical Society 
The main purpose of this paper is to give a similar theorem for Cohen-Macaulay dimension to characterize Cohen-Macaulay local rings of prime characteristic. In Section 3, we shall consider F-finite local rings, and prove the following theorem. (In the following theorem, $\nu(R)$ is an invariant of the local ring $R$, whose definition we will give in Section 3.)

Theorem 3.5. Let $R$ be an F-finite local ring of characteristic p. Then the following conditions are equivalent:

(1) $R$ is Cohen-Macaulay.

(2) $\mathrm{CM}-\operatorname{dim}_{R}{ }^{e} R<\infty$ for all integers $e$.

(3) $\mathrm{CM}-\operatorname{dim}_{R}{ }^{e} R<\infty$ for infinitely many integers e.

(4) $\mathrm{CM}-\operatorname{dim}_{R}{ }^{e} R<\infty$ for some integer e with $p^{e} \geq \nu(R)$.

To prove this theorem, the following lemma will be a key which plays an essential role in proving several other results of this paper.

Lemma 3.2. Let $(R, \mathfrak{m}, k) \rightarrow(S, \mathfrak{n}, l)$ be a local homomorphism of local rings. Suppose that $\mathfrak{m} S \subseteq \mathfrak{n}^{\nu(S)}$. Then, there is an $S$-regular sequence $\mathbf{y}$ such that $k$ is isomorphic to a direct summand of $S /(\mathbf{y})$ as an R-module.

In Section 5, we shall also make a consideration for local rings which are not necessarily F-finite, and prove the following result that generalizes Theorem 3.5

Theorem 5.3. Let $(R, \mathfrak{m}, k)$ be a local ring of characteristic $p$. Then the following conditions are equivalent:

(1) $R$ is Cohen-Macaulay.

(2) There exist a local ring homomorphism $R \rightarrow S$ and a finitely generated $S$-module $N$ satisfying the following two conditions:

i) There is a maximal $R$-regular sequence that is also $N$-regular.

ii) There is an integer e such that $p^{e} \geq \nu(R)$ and $\operatorname{Ext}_{R}^{i}\left({ }^{e} R, N\right)=0$ for any sufficiently large integer $i$.

The organization of this paper is as follows. In Section 2, we recall the definitions and elementary properties of Cohen-Macaulay dimension and Gorenstein dimension without proofs. In Section 3, we shall give proofs of Lemma 3.2 and Theorem 3.5 In Section 4, we make a generalization of a theorem of J. Herzog [16], which gives a sufficient condition for a module to have finite projective dimension or finite injective dimension. Using Lemma 3.2 and a result due to Avramov and Foxby [4], we shall actually refine the result of Herzog in Theorem 4.5 In Section 5, we give a proof of Theorem 5.3 In Section 6, we give a characterization of F-finite Gorenstein local rings. It is similar to Theorem [3.5, but is stated in a slightly stronger form.

\section{Preliminaries}

In this section, we shall recall the definitions of Cohen-Macaulay dimension defined by A. A. Gerko [14 and Gorenstein dimension defined by M. Auslander [1], and state several properties of those dimensions for later use.

Throughout this section, $R$ always denotes a local ring with residue field $k$.

In order to define Cohen-Macaulay dimension and Gorenstein dimension, we begin by observing the notion of semi-dualizing module, which has been studied by H.-B. Foxby [13], E. S. Golod [15], L. W. Christensen [11], and Gerko [14]. (A semi-dualizing module is a $P G$-module which appears in [13], and is called a suitable 
module in [15] and [14.) We denote by $\mathcal{D}^{b}(R)$ the derived category of the category of finitely generated $R$-modules.

Definition. (1) A finitely generated $R$-module $C$ is said to be semi-dualizing if the natural homomorphism $R \rightarrow \operatorname{Hom}_{R}(C, C)$ is an isomorphism and $\operatorname{Ext}_{R}^{i}(C, C)=0$ for $i>0$ (or, equivalently, if the natural morphism $R \rightarrow \operatorname{RHom}_{R}(C, C)$ is an isomorphism in $\left.\mathcal{D}^{b}(R)\right)$.

(2) For a semi-dualizing $R$-module $C$, we say that a finitely generated $R$-module $M$ is totally $C$-reflexive if the natural homomorphism $M \rightarrow \operatorname{Hom}_{R}\left(\operatorname{Hom}_{R}(M, C), C\right)$ is an isomorphism and $\operatorname{Ext}_{R}^{i}(M, C)=\operatorname{Ext}_{R}^{i}\left(\operatorname{Hom}_{R}(M, C), C\right)=0$ for $i>0$.

One should note that the $R$-module $R$ is always a semi-dualizing module.

Semi-dualizing modules satisfy several properties; see the following proposition. For their proofs, see [11] and [13]. Here, for a finitely generated $R$-module $M$, $\mu_{R}(M)$ denotes the minimal number of generators of $M$, and $r_{R}(M)$ denotes the type of $M$, i.e., $\mu_{R}(M)=\operatorname{dim}_{k}\left(M \otimes_{R} k\right)$ and $r_{R}(M)=\operatorname{dim}_{k} \operatorname{Ext}_{R}^{t}(k, M)$, where $t=\operatorname{depth}_{R} M$.

Proposition 2.1. A semi-dualizing $R$-module $C$ has the following properties:

(1) It is faithful.

(2) A sequence of elements of $R$ is $R$-regular if and only if it is $C$-regular. In particular, $\operatorname{depth}_{R} C=\operatorname{depth} R$.

(3) $\mu_{R}(C) r_{R}(C)=r_{R}(R)$.

Now let us state the definitions of Cohen-Macaulay dimension and Gorenstein dimension. We denote by $\Omega_{R}^{n} M$ the $n$-th syzygy module of an $R$-module $M$.

Definition. Let $M$ be a finitely generated $R$-module.

(1) If there is a faithfully flat homomorphism $R \rightarrow S$ of local rings together with a semi-dualizing $S$-module $C$ such that the module $\Omega_{S}^{n}\left(M \otimes_{R} S\right)$ is totally $C$-reflexive, then we say that the Cohen-Macaulay dimension (abbr. CM-dimension) of $M$ is not larger than $n$, and write $\mathrm{CM}-\operatorname{dim}_{R} M \leq n$. If there does not exist such an integer $n$, we say that the CM-dimension of $M$ is infinite, and write $\mathrm{CM}-\operatorname{dim}_{R} M=\infty$.

(2) If the module $\Omega_{R}^{n} M$ is totally $R$-reflexive, then we say that the Gorenstein dimension (abbr. G-dimension) of $M$ is not larger than $n$, and write G-dim ${ }_{R} M \leq n$. If there does not exist such an integer $n$, we say that the G-dimension of $M$ is infinite, and write G- $\operatorname{dim}_{R} M=\infty$.

Remark 2.2. In [14] Gerko defines CM-dimension in two different ways. Compare [14, Definition 3.2] with [14 Definition 3.2']. We have adopted the latter one in the above definition. Since he proves in his paper that they coincide with each other, the following conditions are equivalent for a finitely generated $R$-module $M$.

(1) $\mathrm{CM}-\operatorname{dim}_{R} M<\infty$.

(2) There exist a faithfully flat homomorphism $R \rightarrow S$ and a surjective homomorphism $T \rightarrow S$ of local rings such that G- $\operatorname{dim}_{T} S=\operatorname{grade}_{T} S(<\infty)$ and G- $\operatorname{dim}_{T}\left(M \otimes_{R} S\right)<\infty$.

G-dimension and CM-dimension satisfy many properties, as follows. Notice that these properties are quite similar to those of projective dimension. For their proofs, see [2], [3], [12], [14], [19], and [23]

Proposition 2.3. (1) If $\mathrm{G}$ - $\operatorname{dim}_{R} M<\infty$ for a finitely generated $R$-module $M$, then $\mathrm{G}-\operatorname{dim}_{R} M=\operatorname{depth} R-\operatorname{depth}_{R} M$. 
(2) The following conditions are equivalent:

i) $R$ is Gorenstein.

ii) $\mathrm{G}-\operatorname{dim}_{R} M<\infty$ for any finitely generated $R$-module $M$.

iii) $\mathrm{G}-\operatorname{dim}_{R} k<\infty$.

(3) Let $N$ be a direct summand of an $R$-module $M$. Then $\mathrm{G}$ - $\operatorname{dim}_{R} N<\infty$ whenever $\mathrm{G}-\operatorname{dim}_{R} M<\infty$.

(4) Let $0 \rightarrow L \rightarrow M \rightarrow N \rightarrow 0$ be a short exact sequence of finitely generated $R$-modules. If any two of $L, M, N$ have finite $G$-dimension, then so does the third.

(5) The following conditions are equivalent for a finitely generated $R$-module:

i) $\mathrm{G}-\operatorname{dim}_{R} M<\infty$.

ii) The natural morphism $M \rightarrow \mathbf{R H o m}_{R}\left(\mathbf{R H o m}_{R}(M, R), R\right)$ is an isomorphism in $\mathcal{D}^{b}(R)$.

(6) If CM- $\operatorname{dim}_{R} M<\infty$ for a finitely generated $R$-module $M$, then $\mathrm{CM}-\operatorname{dim}_{R} M$ $=\operatorname{depth} R-\operatorname{depth}_{R} M$.

(7) The following conditions are equivalent:

i) $R$ is Cohen-Macaulay.

ii) $\mathrm{CM}-\operatorname{dim}_{R} M<\infty$ for any finitely generated $R$-module $M$.

iii) $\mathrm{CM}-\operatorname{dim}_{R} k<\infty$.

(8) Let $N$ be a direct summand of a finitely generated $R$-module $M$. Then $\mathrm{CM}-\operatorname{dim}_{R} N<\infty$ whenever CM-dim $R=\infty$.

(9) $\mathrm{CM}-\operatorname{dim}_{R} M \leq \mathrm{G}-\operatorname{dim}_{R} M$ for any finitely generated $R$-module $M$. In particular, a module of finite G-dimension is also of finite CM-dimension.

\section{A characterization of Cohen-Macaulay Rings}

First of all, we introduce an invariant of a local ring.

Definition. Let $R$ be a local ring with maximal ideal $\mathfrak{m}$. Take a maximal $R$ regular sequence $\mathbf{x}=x_{1}, x_{2}, \cdots, x_{t}$, where $t=\operatorname{depth} R$. Then, note that the 0-th local cohomology module $H_{\mathfrak{m}}^{0}(R /(\mathbf{x}))$ of $R /(\mathbf{x})$ with respect to $\mathfrak{m}$ is a nontrivial submodule of $R /(\mathbf{x})$ of finite length; hence it follows from the Artin-Rees lemma that $H_{\mathfrak{m}}^{0}(R /(\mathbf{x})) \cap \mathfrak{m}^{n}(R /(\mathbf{x}))=0$ for any sufficiently large $n$. We define the integer-valued invariant $\nu(R)$ to be the smallest integer $n$ satisfying $H_{\mathfrak{m}}^{0}(R /(\mathbf{x})) \cap$ $\mathfrak{m}^{n}(R /(\mathbf{x}))=0$ for some maximal $R$-regular sequence $\mathbf{x}$.

Remark 3.1. (1) Several invariants similar to the above invariant has been investigated in [17].

(2) Note that, from the definition, $R$ is a regular local ring if and only if $\nu(R)=1$. Furthermore, if $(R, \mathfrak{m})$ is a Cohen-Macaulay local ring, then $\nu(R)$ is the smallest integer $n$ such that $\mathfrak{m}^{n}$ is contained in some parameter ideal of $R$. Hence it is not bigger than the multiplicity of $R$ with respect to $\mathfrak{m}$.

(3) Let $(R, \mathfrak{m}) \rightarrow(S, \mathfrak{n})$ be a faithfully flat homomorphism of local rings. Assume that its closed fiber is artinian, and let $l$ be the smallest integer with $\mathfrak{n}^{l} \subseteq \mathfrak{m} S$. Then we have the inequality $\nu(S) \leq l \cdot \nu(R)$.

In fact, take a maximal $R$-regular sequence $\mathbf{x}$ with $H_{\mathfrak{m}}^{0}(R /(\mathbf{x})) \cap \mathfrak{m}^{\nu(R)}(R /(\mathbf{x}))=$ 0 . Then, since the induced map $R /(\mathbf{x}) \rightarrow S / \mathbf{x} S$ is also faithfully flat, it is easy to 
see that $H_{\mathfrak{n}}^{0}(S / \mathbf{x} S)=H_{\mathfrak{m}}^{0}(R /(\mathbf{x}))(S / \mathbf{x} S)$ and

$$
\begin{aligned}
H_{\mathfrak{n}}^{0}(S / \mathbf{x} S) \cap \mathfrak{n}^{l \cdot \nu(R)}(S / \mathbf{x} S) & \subseteq H_{\mathfrak{m}}^{0}(R /(\mathbf{x}))(S / \mathbf{x} S) \cap \mathfrak{m}^{\nu(R)}(S / \mathbf{x} S) \\
& =\left(H_{\mathfrak{m}}^{0}(R /(\mathbf{x})) \cap \mathfrak{m}^{\nu(R)}(R /(\mathbf{x}))\right)(S / \mathbf{x} S) \\
& =0 .
\end{aligned}
$$

Hence we have $\nu(S) \leq l \cdot \nu(R)$.

Now we shall prove the main lemma of this section. It is rather easy to prove the lemma, but it has a lot of applications, as we shall see later.

Lemma 3.2. Let $(R, \mathfrak{m}, k) \rightarrow(S, \mathfrak{n}, l)$ be a homomorphism of local rings. Suppose that $\mathfrak{m} S \subseteq \mathfrak{n}^{\nu(S)}$. Then, there is an $S$-regular sequence $\mathbf{y}$ such that $k$ is isomorphic to a direct summand of $S /(\mathbf{y})$ as an $R$-module.

Proof. Take a maximal $S$-regular sequence y with $H_{\mathfrak{n}}^{0}(S /(\mathbf{y})) \cap \mathfrak{n}^{\nu(S)}(S /(\mathbf{y}))=0$. Set $\bar{S}=S /(\mathbf{y})$, and let $\theta: H_{\mathfrak{n}}^{0}(\bar{S}) \rightarrow \bar{S}$ and $\pi: \bar{S} \rightarrow \bar{S} / \mathfrak{m} \bar{S}$ be natural homomorphisms. Since $\mathfrak{m} S \subseteq \mathfrak{n}^{\nu(S)}$, we have $H_{\mathfrak{n}}^{0}(\bar{S}) \cap \mathfrak{m} \bar{S}=0$. Hence the composite map $\pi \theta$ is injective. Note that $\bar{S} / \mathfrak{m} \bar{S}$ is a $k$-vector space; hence so is $H_{\mathfrak{n}}^{0}(\bar{S})$. Therefore $\pi \theta$ is a split-monomorphism of $R$-modules; hence so is $\theta$. Since $H_{\mathfrak{n}}^{0}(\bar{S})$ is a non-zero $k$-vector space, the assertion follows.

Applying the above lemma to the Frobenius map, one obtains the following result for local rings of prime characteristic.

Corollary 3.3. Let $(R, \mathfrak{m}, k)$ be a local ring of characteristic $p$, and let $e$ be an integer with $p^{e} \geq \nu(R)$. Then $k$ is isomorphic to a direct summand of ${ }^{e} R /(\mathbf{x})$ as an $R$-module for some maximal ${ }^{e} R$-regular sequence $\mathbf{x}$ in ${ }^{e} \mathfrak{m}$.

Proof. Let $a_{1}, a_{2}, \cdots, a_{r}$ be a system of generators of $\mathfrak{m}$. Then the ideal $\mathfrak{m}^{e} R$ of ${ }^{e} R$ is generated by $a_{1}^{p^{e}}, a_{2}^{p^{e}}, \cdots, a_{r}^{p^{e}}$. On the other hand, the elements $a_{1}, a_{2}, \cdots, a_{r}$ in ${ }^{e} R$ generate the ideal ${ }^{e} \mathfrak{m}$. Since $p^{e} \geq \nu(R)=\nu\left({ }^{e} R\right)$, one has $\mathfrak{m}^{e} R \subseteq\left({ }^{e} \mathfrak{m}\right)^{\nu\left({ }^{e} R\right)}$. Thus the assertion follows from Lemma 3.2 .

Corollary 3.4. Let $\mathcal{P}$ be a property of local rings, and let $(R, \mathfrak{m}, k)$ be an F-finite local ring of characteristic $p$. Consider a numerical invariant $\lambda$ of a finitely generated $R$-module (that is, $\lambda(0)=-\infty$ and $\lambda(X) \in \mathbf{N} \cup\{\infty\}$ for a finitely generated $R$-module $X)$ satisfying the following conditions:

a) Let $R \rightarrow S$ be a finite homomorphism of local rings, and let $y \in S$ be a non-zero-divisor on $S$. If $\lambda(S)<\infty$, then $\lambda(S /(y))<\infty$.

b) Let $N$ be a direct summand of a finitely generated $R$-module $M$. If $\lambda(M)<$ $\infty$, then $\lambda(N)<\infty$.

c) If $\lambda(k)<\infty$, then $R$ satisfies $\mathcal{P}$.

Under these circumstances, suppose that $\lambda\left({ }^{e} R\right)<\infty$ for some integer e with $p^{e} \geq \nu(R)$. Then $R$ satisfies $\mathcal{P}$.

Before proving this corollary, we should note that there are many numerical invariants satisfying the conditions a)-c) for certain properties of local rings. For instance, projective dimension satisfies them for the regular property. We will give several examples after the proof of this corollary, and again after Theorem 3.5.

Proof of Corollary 3.4 Assume that $\lambda\left({ }^{e} R\right)<\infty$ for some integer $e$ with $p^{e} \geq \nu(R)$. We have from Corollary 3.3 that $k$ is a direct summand of ${ }^{e} R /(\mathbf{x})$ as an $R$-module, 
for some ${ }^{e} R$-sequence $\mathbf{x}$. It follows from condition a) that $\lambda\left({ }^{e} R /(\mathbf{x})\right)<\infty$. Hence we see that $\lambda(k)<\infty$ by b), and $R$ satisfies property $\mathcal{P}$ by c).

Now we observe that CM-dimension is a numerical invariant satisfying conditions a)-c) in the above corollary for the Cohen-Macaulay property. Let us take CM-dimension as $\lambda$, and the Cohen-Macaulay property as $\mathcal{P}$ in the corollary. Then it follows from Proposition 2.3.7 and 2.3.8 that CM-dimension satisfies conditions b) and c) for the Cohen-Macaulay property. Furthermore, it is easy to see that CM-dimension also satisfies the condition a). Indeed, let $R \rightarrow S$ be a finite homomorphism of local rings, and let $y \in S$ be a non-zero-divisor on $S$. Suppose that $\mathrm{CM}-\operatorname{dim}_{R} S<\infty$. Then, by Remark 2.2, there exist a faithfully flat homomorphism $R \rightarrow R^{\prime}$ and a surjective homomorphism $T \rightarrow R^{\prime}$ of local rings such that G- $\operatorname{dim}_{T} R^{\prime}=\operatorname{grade}_{T} R^{\prime}<\infty$ and G-dim$T\left(S \otimes_{R} R^{\prime}\right)<\infty$. On the other hand, we have a short exact sequence

$$
0 \rightarrow S \stackrel{y}{\rightarrow} S \rightarrow S /(y) \rightarrow 0 .
$$

Since $R^{\prime}$ is faithfully flat, the tensored sequence

$$
0 \rightarrow S \otimes_{R} R^{\prime} \stackrel{y}{\rightarrow} S \otimes_{R} R^{\prime} \rightarrow(S /(y)) \otimes_{R} R^{\prime} \rightarrow 0
$$

is also exact. Hence we see from Proposition 2.34 that G-dim $\operatorname{dim}_{T}\left((S /(y)) \otimes_{R} R^{\prime}\right)<\infty$, and we have $\mathrm{CM}-\operatorname{dim}_{R} S /(y)<\infty$, as desired.

Corollary 3.4 and the above remark induce the implication $(4) \Rightarrow(1)$ in the following theorem. The implications $(2) \Rightarrow(3)$ and $(3) \Rightarrow(4)$ are obvious, while $(1) \Rightarrow(2)$ follows from Proposition 2.37 .

Theorem 3.5. Let $R$ be an F-finite local ring of characteristic $p$. Then the following conditions are equivalent:

(1) $R$ is Cohen-Macaulay.

(2) $\mathrm{CM}-\operatorname{dim}_{R}{ }^{e} R<\infty$ for all integers $e$.

(3) $\mathrm{CM}-\operatorname{dim}_{R}{ }^{e} R<\infty$ for infinitely many integers e.

(4) $\mathrm{CM}-\operatorname{dim}_{R}{ }^{e} R<\infty$ for some integer e with $p^{e} \geq \nu(R)$.

Projective dimension, CI-dimension, and G-dimension also satisfy conditions a)c) in Corollary 3.4 for the associated properties of local rings. Hence an F-finite local ring $R$ of characteristic $p$ is a regular ring (resp. a complete intersection, a Gorenstein ring) if ${ }^{e} R$ has finite projective dimension (resp. finite CI-dimension, finite G-dimension) for some integer $e$ with $p^{e} \geq \nu(R)$. Compare this with Theorem 1.1, Theorem 1.2, and Theorem 6.2

Moreover, since upper G-dimension $\mathrm{G}^{*}$-dim and lower CI-dimension $\mathrm{CI}_{*}$-dim also satisfy those conditions, an F-finite local ring $R$ is a Gorenstein ring (resp. a complete intersection) if ${ }^{e} R$ has finite upper G-dimension (resp. finite lower CIdimension) for some integer $e$ with $p^{e} \geq \nu(R)$. (For the details of these homological dimensions, see [3], 14], and [22].)

We end this section by proposing a natural conjecture.

Conjecture. Let $R$ be an F-finite local ring. Suppose that the $R$-module ${ }^{e} R$ has finite CM-dimension for some integer e $>0$. Then $R$ would be Cohen-Macaulay.

The authors believe that this is true, but have no proof of it at this moment. Compare this with Theorem 6.2 to see that the corresponding statement about G-dimension is true. 


\section{A generalization of Herzog's theorem}

J. Herzog proved in his paper [16] the following theorem as a generalization of a theorem due to E. Kunz [18].

Theorem 4.1 (Herzog). Let $R$ be a local ring of characteristic $p$, and let $M$ be a finitely generated $R$-module.

(1) If $\operatorname{Tor}_{i}^{R}\left(M,{ }^{e} R\right)=0$ for any $i>0$ and infinitely many $e$, then $\operatorname{pd}_{R} M<\infty$.

(2) Suppose that $R$ is F-finite. If $\operatorname{Ext}_{R}^{i}\left({ }^{e} R, M\right)=0$ for any $i>0$ and infinitely many $e$, then $\operatorname{id}_{R} M<\infty$.

According to the method of Herzog, the first assertion of this theorem is proved by observing the structure of the complex $F_{\bullet} \otimes_{R}{ }^{e} R$, where $F_{\bullet}$ is a minimal $R$-free resolution of the module $M$, and the second assertion is proved by considering the Matlis duality and using the first assertion. However, Lemma 3.2 actually makes it easy for us to prove the assertions of Herzog's theorem in a more general setting.

To do this, we introduce the following result of Avramov and Foxby.

Lemma $4.2([4$, Proposition 5.5]). Let $(R, \mathfrak{m}, k) \rightarrow(S, \mathfrak{n}, l)$ be a local homomorphism of local rings, and let $N$ be a finitely generated $S$-module. Then:

(1) $\operatorname{fd}_{R} N=\sup \left\{i \mid \operatorname{Tor}_{i}^{R}(N, k) \neq 0\right\}$.

(2) $\operatorname{id}_{R} N=\sup \left\{i \mid \operatorname{Ext}_{R}^{i}(k, N) \neq 0\right\}$.

Combining this lemma with Lemma 3.2 we obtain the following proposition.

Proposition 4.3. Let $(R, \mathfrak{m}, k) \rightarrow\left(R^{\prime}, \mathfrak{m}^{\prime}, k^{\prime}\right)$ and $(R, \mathfrak{m}, k) \rightarrow(S, \mathfrak{n}, l)$ be local homomorphisms of local rings, and let $N$ be a finitely generated $S$-module. Suppose that $\mathfrak{m} R^{\prime} \subseteq \mathfrak{m}^{\prime \nu\left(R^{\prime}\right)}$.

(1) If $\operatorname{Tor}_{i}^{R}\left(N, R^{\prime}\right)=0$ for any sufficiently large integer $i$, then $\operatorname{fd}_{R} N<\infty$.

(2) If $\operatorname{Ext}_{R}^{i}\left(R^{\prime}, N\right)=0$ for any sufficiently large integer $i$, then $\operatorname{id}_{R} N<\infty$.

Proof. First of all, we note from Lemma 3.2 that there exists a maximal $R^{\prime}$-regular sequence $\mathbf{x}$ such that $k$ is a direct summand of $R^{\prime} /(\mathbf{x})$ as an $R$-module. From the assumption we easily see that $\operatorname{Tor}_{i}^{R}\left(N, R^{\prime} /(\mathbf{x})\right)=0$ or $\operatorname{Ext}_{R}^{i}\left(R^{\prime} /(\mathbf{x}), N\right)=0$ for $i \gg 0$. Hence we have $\operatorname{Tor}_{i}^{R}(N, k)=0$ and $\operatorname{Ext}_{R}^{i}(k, N)=0$ for $i \gg 0$. Thus the proposition follows from Lemma 4.2

Remark 4.4. Let $(R, \mathfrak{m}) \rightarrow(S, \mathfrak{n})$ be a homomorphism of local rings such that $\mathfrak{m} S \subseteq \mathfrak{n}^{\nu(S)}$ and $\operatorname{fd}_{R} S<\infty$. Then $R$ is regular.

In fact, since $\operatorname{Tor}_{i}^{R}(k, S)=0$ for $i \gg 0$, it follows from Proposition 4.3 that $\left(\operatorname{pd}_{R} k=\right) \mathrm{fd}_{R} k<\infty$. Hence the local ring $R$ is regular.

It is easy to see that the next theorem follows from Proposition 4.3. Notice that the second assertion in the theorem holds without F-finiteness of the ring $R$.

Theorem 4.5. Let $R \rightarrow S$ be a local homomorphism of local rings, and let $N$ be a finitely generated $S$-module. Suppose that $R$ has characteristic $p$. Let $e$ be an integer with $p^{e} \geq \nu(R)$.

(1) If $\operatorname{Tor}_{i}^{R}\left(N,{ }^{e} R\right)=0$ for any sufficiently large integer $i$, then $\operatorname{fd}_{R} N<\infty$.

(2) If $\operatorname{Ext}_{R}^{i}\left({ }^{e} R, N\right)=0$ for any sufficiently large integer $i$, then $\operatorname{id}_{R} N<\infty$.

Needless to say, this theorem implies Theorem 4.1] 


\section{Another Characterization OF COHEN-MACAulay Rings}

Let $R$ be a local ring of characteristic $p$. In Section 3 we have given necessary and sufficient conditions for $R$ to be Cohen-Macaulay in terms of the CM-dimension of the $R$-module ${ }^{e} R$, in the case that the $\operatorname{ring} R$ is F-finite. In this section, we would like to characterize Cohen-Macaulay local rings of prime characteristic which are not necessarily F-finite.

Proposition 5.1. Let $R$ be a local ring with maximal ideal $\mathfrak{m}$. Assume that there exists a non-zero injective $R$-module which is separated in its $\mathfrak{m}$-adic topology. Then $R$ is artinian.

Proof. Let $M$ be a non-zero injective $R$-module which is $\mathfrak{m}$-adically separated. Since any submodule of a separated module is also separated, we may assume that $M$ is the injective hull $E_{R}(R / \mathfrak{p})$ of $R / \mathfrak{p}$ for some prime ideal $\mathfrak{p}$ of $R$. Then, since $M=E_{R}(R / \mathfrak{p})$ is an $R_{\mathfrak{p}}$-module and $M$ is $\mathfrak{m}$-adically separated, we must have $\mathfrak{p}=\mathfrak{m}$. Thus $M$ is an artinian $R$-module. Then $\bigcap_{n} \mathfrak{m}^{n} M=0$ implies $\mathfrak{m}^{n} M=0$ for some integer $n$. Since the module $\operatorname{End}_{R}(M)$ is isomorphic to the $\mathfrak{m}$-adic completion $\widehat{R}$ of $R$, we finally have that $\mathfrak{m}^{n}=0$, and hence $R$ is an artinian ring.

In the case when the module $N$ is finitely generated over the ring $R$, the following proposition is a well-known result due to H. Bass 8].

Proposition 5.2. Let $(R, \mathfrak{m}, k) \rightarrow(S, \mathfrak{n}, l)$ be a local homomorphism of local rings, and let $N$ be a non-zero finitely generated $S$-module with $\operatorname{id}_{R} N<\infty$. Then $\operatorname{id}_{R} N=$ $\operatorname{depth} R$.

Proof. Set $s=\operatorname{id}_{R} N$ and $t=\operatorname{depth} R$, and take a maximal $R$-regular sequence $\mathbf{x}=x_{1}, x_{2}, \cdots, x_{t}$. Since depth $R /(\mathbf{x})=0$, there is a short exact sequence $0 \rightarrow$ $k \rightarrow R /(\mathbf{x}) \rightarrow C \rightarrow 0$ of $R$-modules. Hence we have an exact sequence

$$
\operatorname{Ext}_{R}^{s}(R /(\mathbf{x}), N) \rightarrow \operatorname{Ext}_{R}^{s}(k, N) \rightarrow \operatorname{Ext}_{R}^{s+1}(C, N)=0
$$

Lemma 4.2 implies that $\operatorname{Ext}_{R}^{s}(k, N) \neq 0$; hence $\operatorname{Ext}_{R}^{s}(R /(\mathbf{x}), N) \neq 0$. Since the Koszul complex $K_{\bullet}(\mathbf{x})$ of $\mathbf{x}$ over $R$ is an $R$-free resolution of $R /(\mathbf{x})$ of length $t$, we see that $s \leq t$. On the other hand, it follows from Nakayama's lemma that $\operatorname{Ext}_{R}^{t}(R /(\mathbf{x}), N) \cong N / \mathbf{x} N \neq 0$. Therefore $s=t$, as desired.

Now we are ready to prove a theorem which characterizes the Cohen-Macaulay property for local rings of prime characteristic that are not necessarily F-finite.

Theorem 5.3. Let $(R, \mathfrak{m}, k)$ be a local ring of characteristic $p$. Then the following conditions are equivalent:

(1) $R$ is Cohen-Macaulay.

(2) There exist a local ring homomorphism $R \rightarrow S$ and a finitely generated $S$-module $N$ satisfying the following two conditions.

i) There is a maximal $R$-regular sequence that is also $N$-regular.

ii) There is an integer e such that $p^{e} \geq \nu(R)$ and $\operatorname{Ext}_{R}^{i}\left({ }^{e} R, N\right)=0$ for any sufficiently large integer $i$.

Proof. $(1) \Rightarrow(2)$ Let $S$ be the m-adic completion of the Cohen-Macaulay local ring $R$, and let $N$ be the canonical module of $S$. Note that $S$ is faithfully flat over $R$, and that $N$ is a maximal Cohen-Macaulay $S$-module. Hence it is easy to see that every $R$-regular sequence is also $S$-regular, and that every $S$-regular sequence 
is also $N$-regular. Noting that $N$ is of finite injective dimension over $S$, one has $\operatorname{Ext}_{R}^{i}\left({ }^{e} R, N\right) \cong \operatorname{Ext}_{S}^{i}\left({ }^{e} R \otimes_{R} S, N\right)=0$ for any $e>0$ and $i \gg 0$.

$(2) \Rightarrow(1)$ Set $t=\operatorname{depth} R$. By the assumption, we have a sequence $\mathbf{x}=$ $x_{1}, x_{2}, \cdots, x_{t}$ of elements of $R$ which is both $R$-regular and $N$-regular. Theorem 4.5 and Proposition 5.2 imply that $\operatorname{id}_{R} N=t$. In particular, $\operatorname{Ext}_{R}^{i}(k, N)=0$ for $i>t$. Hence we also have $\operatorname{Ext}_{R /(\mathbf{x})}^{i}(k, N / \mathbf{x} N)=0$ for $i>0$. Thus it follows from Lemma 4.2 that $\operatorname{id}_{R /(\mathbf{x})} N / \mathbf{x} N=0$, i.e., $N / \mathbf{x} N$ is $R /(\mathbf{x})$-injective. On the other hand, since the induced homomorphism $R /(\mathbf{x}) \rightarrow S / \mathrm{x} S$ is local and since $N / \mathrm{x} N$ is a finitely generated $S / \mathbf{x} S$-module, $N / \mathbf{x} N$ is $\mathfrak{m} /(\mathbf{x})$-adically separated. Therefore it follows from Proposition 5.1 that $R /(\mathbf{x})$ is artinian, and so $R$ is Cohen-Macaulay.

As a corollary of the above theorem, we obtain the characterization of F-finite Cohen-Macaulay local rings as it is stated in Section 3. We give here an alternative proof of the implication (4) $\Rightarrow(1)$ of Theorem 3.5 by using the above theorem.

Alternative proof of Theorem $3.5(4) \Rightarrow(1)$. Since $\mathrm{CM}-\operatorname{dim}_{R}{ }^{e} R<\infty$, one has CM- $\operatorname{dim}_{R}{ }^{e} R=\operatorname{depth} R-\operatorname{depth}_{R}{ }^{e} R$ by Proposition 2.3.6. Noting that a sequence $x_{1}, x_{2}, \cdots, x_{n}$ of elements of $R$ is ${ }^{e} R$-regular if and only if $x_{1}^{p^{e}}, x_{2}^{p^{e}}, \cdots, x_{n}^{p^{e}}$ is an $R$-regular sequence, one sees that $\operatorname{depth}_{R}{ }^{e} R=\operatorname{depth} R$; hence CM-dim ${ }_{R}^{e} R=0$. So it follows from the definition that there exist a faithfully flat homomorphism $R \rightarrow S$ of local rings and a semi-dualizing $S$-module $C$ such that $\operatorname{Ext}_{S}^{i}\left({ }^{e} R \otimes_{R} S, C\right)=0$ for $i>0$. Since $S$ is faithfully flat over $R$, one has from Proposition 2.1,2 that every $R$-regular sequence is $C$-regular, and $\operatorname{Ext}_{R}^{i}\left({ }^{e} R, C\right) \cong \operatorname{Ext}_{S}^{i}\left({ }^{e} R \otimes_{R} S, C\right)=0$ for $i>0$. Thus Theorem 5.3 implies that $R$ is Cohen-Macaulay.

\section{A characterization of Gorenstein Rings}

We shall prove in this section a result concerning G-dimension, which is similar to Theorem 3.5 but in a slightly stronger form. We begin by stating a proposition that follows directly from Theorem 4.52 .

Proposition 6.1. Let $R$ be a local ring of characteristic $p$. Then the following conditions are equivalent:

(1) $R$ is Gorenstein.

(2) There is an integer e such that $p^{e} \geq \nu(R)$ and $\operatorname{Ext}_{R}^{i}\left({ }^{e} R, R\right)=0$ for any sufficiently large integer $i$.

Now we can prove the main result of this section.

Theorem 6.2. The following conditions are equivalent for an F-finite local ring $R$ of prime characteristic $p$ :

(1) $R$ is Gorenstein.

(2) $\mathrm{G}-\operatorname{dim}_{R}{ }^{e} R<\infty$ for every integer $e>0$.

(3) $\mathrm{G}-\operatorname{dim}_{R}{ }^{e} R<\infty$ for infinitely many integers $e>0$.

(4) $\mathrm{G}-\operatorname{dim}_{R}{ }^{e} R<\infty$ for some integer e with $p^{e} \geq \nu(R)$.

(5) $\mathrm{G}-\operatorname{dim}_{R}{ }^{e} R<\infty$ for some integer e $>0$.

Proof. The implications $(1) \Rightarrow(2) \Rightarrow(3) \Rightarrow(4) \Rightarrow(5)$ are obvious from Proposition 2.3. 2, and the implication (4) $\Rightarrow(1)$ follows from Proposition 6.1]

It remains to prove the implication $(5) \Rightarrow(4)$. For this, it is enough to show the following claim. 
Claim. If G- $\operatorname{dim}_{R}{ }^{e} R<\infty$ for some integer $e$, then $\mathrm{G}-\operatorname{dim}_{R}{ }^{2 e} R<\infty$.

Suppose that $\mathrm{G}-\operatorname{dim}_{R}{ }^{e} R<\infty$ for some integer $e$. Then we have G- $\operatorname{dim}_{R}{ }^{e} R=$ $\operatorname{depth} R-\operatorname{depth}_{R}{ }^{e} R=0$, and hence $\operatorname{Hom}_{R}\left({ }^{e} R, R\right) \cong \mathbf{R} \operatorname{Hom}_{R}\left({ }^{e} R, R\right)$ in $\mathcal{D}^{b}(R)$. Now put $C=\operatorname{Hom}_{R}\left({ }^{e} R, R\right)$, and we see from Proposition 2.35 that

$$
\begin{aligned}
\mathbf{R H o m}_{e}(C, C) & \cong \mathbf{R H o m}_{e}\left(\mathbf{R H o m}_{R}\left({ }^{e} R, R\right), \mathbf{R} \operatorname{Hom}_{R}\left({ }^{e} R, R\right)\right) \\
& \cong \mathbf{R H o m}_{R}\left(\mathbf{R H o m} R\left({ }^{e} R, R\right), R\right) \\
& \cong{ }^{e} R .
\end{aligned}
$$

Therefore $C$ is a semi-dualizing ${ }^{e} R$-module.

We would like to show that $C$ is isomorphic to ${ }^{e} R$ as an ${ }^{e} R$-module. To do this, let ${ }^{e} k$ be the residue field of ${ }^{e} R$, and put $t=\operatorname{depth} R$. Since $\mathbf{R H o m}_{R}\left({ }^{e} k, C\right) \cong$ $\mathbf{R H o m}_{R}\left({ }^{e} k, \mathbf{R H o m}_{R}\left({ }^{e} R, R\right)\right) \cong \mathbf{R H o m}_{R}\left({ }^{e} k, R\right)$, we have

$$
\operatorname{Ext}_{e}^{t}\left({ }^{e} k, C\right) \cong \operatorname{Ext}_{R}^{t}\left({ }^{e} k, R\right) \text {. }
$$

Note that $\operatorname{depth}_{e_{R}} C=\operatorname{depth}^{e} R=t$ by Proposition 2.1.2. Hence, comparing the $k$-dimension of the two sides of the above isomorphism, we have

$$
r_{e}(C) \cdot \operatorname{dim}_{k}{ }^{e} k=\operatorname{dim}_{k} \operatorname{Ext}_{e}^{t}\left({ }^{e} k, C\right)=\operatorname{dim}_{k} \operatorname{Ext}_{R}^{t}\left({ }^{e} k, R\right)=r_{R}(R) \cdot \operatorname{dim}_{k}{ }^{e} k .
$$

Therefore we obtain $r_{e}(C)=r_{R}(R)=r_{e}\left({ }^{e} R\right)$. It then follows from Proposition 2.1.3 that $\mu_{e}(C)=1$, that is, $C$ is a cyclic ${ }^{e} R$-module. But Proposition 2.11 implies that $C \cong{ }^{e} R$, as desired.

Since we have shown that there is an isomorphism $\mathbf{R H o m}_{R}\left({ }^{e} R, R\right) \cong{ }^{e} R$ in $\mathcal{D}^{b}\left({ }^{e} R\right)$, we have ismorphisms

$$
\begin{aligned}
\mathbf{R H o m}_{R}\left(X,{ }^{e} R\right) & \cong \mathbf{R H o m}_{e}\left(X, \mathbf{R H o m}_{R}\left({ }^{e} R, R\right)\right) \\
& \cong \mathbf{R H o m}_{R}(X, R)
\end{aligned}
$$

for any obeject $X$ in $\mathcal{D}\left({ }^{e} R\right)$. Thus we have an isomorphism

$$
\mathbf{R H o m}_{R}\left(\mathbf{R H o m}_{e_{R}}\left({ }^{2 e} R,{ }^{e} R\right),{ }^{e} R\right) \cong \mathbf{R H o m}_{R}\left(\mathbf{R H o m}_{R}\left({ }^{2 e} R, R\right), R\right) .
$$

Noting that G- $\operatorname{dim}_{e^{2}}{ }^{2 e} R=\mathrm{G}-\operatorname{dim}_{e_{R}}{ }^{e}\left({ }^{e} R\right)=\mathrm{G}-\operatorname{dim}_{R}{ }^{e} R<\infty$, we see from Proposition 2.3.5 that the left hand side is isomorphic to ${ }^{2 e} R$. Then it follows from Proposition 2.3.5 again that G- $\operatorname{dim}_{R}^{2 e} R<\infty$, and the proof is completed.

\section{ACKNOWLEDGMENTS}

The authors wish to express their deep gratitude to Professor Kohji Yanagawa and the anonymous referee for their valuable comments on the first version of the paper.

\section{REFERENCES}

[1] M. Auslander, Anneaux de Gorenstein, et torsion en algèbre commutative, Séminaire d'algèbre commutative dirigé par P. Samuel, Secrétariat mathématique, Paris, 1967. MR 37:1435

[2] M. Auslander and M. Bridger, Stable module theory, Memoirs of the American Mathematical Society, No. 94, 1969. MR 42:4580

[3] L. L. Avramov, Homological dimensions and related invariants of modules over local rings, Proceedings of the 9th International Conference on Representations of Algebras (Beijing, 2000), to appear.

[4] L. L. Avramov and H.-B. Foxby, Homological dimensions of unbounded complexes, J. Pure Appl. Algebra 71 (1991), no. 2-3, 129-155. MR 93g:18017

[5] L. L. Avramov, H.-B. Foxby, and B. Herzog, Structure of local homomorphisms, J. Algebra 164 (1994), no. 1, 124-145. MR 95f:13029 
[6] L. L. Avramov, V. N. Gasharov, and I. V. Peeva, Complete intersection dimension, Inst. Hautes Études Sci. Publ. Math. No. 86 (1997), 67-114 (1998). MR 99c:13033

[7] T. Araya, R. Takahashi, and Y. Yoshino, Homological invariants associated to semidualizing bimodules, Preprint 2002.

[8] H. Bass, On the ubiquity of Gorenstein rings. Math. Z. 82 (1963), 8-28. MR 27:3669

[9] A. Blanco and J. MAJADAs, Sur les morphismes d'intersection complète en caractéristique p, J. Algebra 208 (1998), no. 1, 35-42. MR 99i:13023

[10] W. Bruns and J. Herzog, Cohen-Macaulay rings, revised version, Cambridge University Press, 1998. MR 95h:13020 (1st ed.)

[11] L. W. Christensen, Semi-dualizing complexes and their Auslander categories, Trans. Amer. Math. Soc. 353 (2001), no. 5, 1839-1883. MR 2002a:13017

[12] L. W. Christensen, Gorenstein dimensions, Lecture Notes in Mathematics, 1747, SpringerVerlag, Berlin, 2000. MR 2002e:13032

[13] H.-B. Foxby, Gorenstein modules and related modules, Math. Scand. 31 (1972), 267-284 (1973). MR 48:6094

[14] A. A. Gerko, On homological dimensions, Mat. Sb. 192 (2001), no. 8, 79-94; translation in Sb. Math. 192 (2001), no. 7-8, 1165-1179. MR 2002h:13024

[15] E. S. Golod, G-dimension and generalized perfect ideals, Trudy Mat. Inst. Steklov. 165 (1984), 62-66; English transl., Proc. Steklov Inst. Math. 1985, no. 3 (164), 67-71. MR 85m:13011

[16] J. Herzog, Ringe der Charakteristik $p$ und Frobeniusfunktoren, Math. Z. 140 (1974), 67-78. MR 50:4569

[17] J. KoH and K. LeE, New invariants of Noetherian local rings, J. Algebra 235 (2001), no. 2, 431-452. MR 2002a:13022

[18] E. Kunz, Characterizations of regular local rings of characteristic p, Amer. J. Math. 91 (1969), 772-784. MR 40:5609

[19] V. MAŞEK, Gorenstein dimension and torsion of modules over commutative noetherian rings, Comm. Algebra 28 (2000), no. 12, 5783-5811. MR 2001k:13025

[20] C. Peskine and L. SzPiro, Dimension projective finie et cohomologie locale. Applications à la démonstration de conjectures de M. Auslander, H. Bass et A. Grothendieck, Inst. Hautes Études Sci. Publ. Math. No. 42 (1973), 47-119. MR 51:10330

[21] A. G. Rodicio, On a result of Avramov, Manuscripta Math. 62 (1988), no. 2, 181-185. MR $89 \mathrm{k}: 13014$

[22] O. Veliche, Construction of modules with finite homological dimensions, J. Algebra 250 (2002), no. 2, 427-449. MR 2003e:13023

[23] S. Yassemi, G-dimension, Math. Scand. 77 (1995), no. 2, 161-174. MR 97d:13017

Graduate School of Natural Science and Technology, Okayama University, Okayama 700-8530, JAPAN

Current address: Faculty of Science, Okayama University, Okayama 700-8530, Japan

E-mail address: takahasi@math.okayama-u.ac.jp

FaCulty of Science, OKayama University, OKayama 700-8530, Japan

E-mail address: yoshino@math.okayama-u.ac.jp 